\title{
¿Es el metro ligero más atractivo que el autobús de alto nivel de servicio?
}

\author{
Alfonso Orro Arcay \\ Profesor Titular de Universidad, Grupo de Ferrocarriles y Transportes, Universidad de A \\ Coruña, España \\ Javier Anta Álvarez \\ Investigador, Grupo de Ferrocarriles y Transportes, Universidad de A Coruña, España \\ Emilio Conles Barrera \\ Investigador, Grupo de Ferrocarriles y Transportes, Universidad de A Coruña, España

\section{Margarita Novales Ordax} \\ Profesora Titular de Universidad, Grupo de Ferrocarriles y Transportes, Universidad de A \\ Coruña, España
}

\section{RESUMEN}

A la hora de realizar una inversión en un nuevo sistema de capacidad intermedia (SCI) en el campo del transporte público urbano y metropolitano, en muchas ocasiones la elección de implantar un metro ligero se ha justificado en una percepción, bastante generalizada, de que los sistemas ferroviarios tenían mayor potencial de captación de viajeros que sistemas equivalentes basados en autobuses. Esto es lo que se ha venido a denominar como rail factor.

En la investigación que se presenta se ha estudiado la existencia de este factor mediante una amplia campaña de encuestas de elección en preferencias reveladas y declaradas, desarrollada en ciudades españolas y francesas con distinta presencia de SCI. Tras la observación de más de 28000 situaciones de elección, se han desarrollado modelos de comportamiento para las diferentes ciudades, empleando modelos logit multinomial, logit jerárquico y logit mixto. Además de variables explicativas tradicionalmente consideradas en el estudio del reparto modal, se han tenido en cuenta otros factores como la experiencia previa de los usuarios en el empleo del sistema de transporte público, la influencia de las elecciones reveladas en las elecciones declaradas o la calidad del servicio de transporte público.

Se ha constatado que en algunos casos existía esa preferencia inherente por el metro ligero, pero que en otros la preferencia de los encuestados era por el autobús de alto nivel de servicio. Finalmente, se ha concluido que no puede afirmarse, de forma general, la existencia de un mayor potencial de captación por parte de los modos ferroviarios respecto a los basados en el autobús, siendo necesaria la realización de encuestas en las circunstancias locales para evaluar el potencial de captación real de cada caso particular. 


\section{INTRODUCCIÓN}

A lo largo de las últimas décadas, han sido numerosas las ciudades que han optado por implantar sistemas de transporte de capacidad intermedia (SCI). Los ejemplos de SCI más habituales son el metro ligero (Light Rail Transit, LRT) y el autobús de alto nivel de servicio (Bus with High Level of Service, BHLS). Para un análisis de las características comunes y las diferencias entre BHLS y LRT puede consultarse Novales, Orro, Conles y Anta (2014). Se ha observado, especialmente en la última década del siglo XX y la primera del siglo XXI, que el LRT ha sido una opción prioritaria sobre el BHLS a la hora de realizar una nueva implantación de SCI. Entre los factores que han explicado este hecho puede encontrarse una suposición de que los modos ferroviarios eran más atractivos que los autobuses. Sin embargo, estudios realizados tras la implantación de algunos sistemas ferroviarios mostraron que, en ocasiones, se había sobreestimado su capacidad de captación de viajeros (Pickrell, 1992).

Esta preferencia por los modos ferroviarios ha sido denominada de diferentes formas, como por ejemplo rail factor (Scherer, 2011), rail bonus (Axhausen, Haupt, Fell y Heidl, 2001) o tram bonus (Bunschoten, 2012). Independientemente de la denominación empleada para diferenciar a los sistemas de transporte, en todos los casos se alude a una preferencia inherente hacia los modos ferroviarios por resultar más atractivos o tener una mejor imagen que los sistemas de transporte basados en el autobús. Es decir, que ante la posibilidad de elegir bien un autobús o bien un modo ferroviario (como por ejemplo un tranvía, un metro ligero, etc.) con las mismas condiciones de nivel de servicio, la imagen percibida de este último influirá significativa y positivamente en la elección del mismo. La cuantificación, o la existencia, de este rail factor han sido debatidas en la literatura, sin que exista una conclusión definitiva. En este trabajo se ha buscado analizar la percepción de ambos tipos de sistemas de transporte mediante encuestas de preferencias realizadas en distintas localizaciones de España y Francia. En la presente investigación se ha considerado la siguiente definición para el rail factor:

El rail factor es un fenómeno que consiste en la existencia de una preferencia inherente hacia los sistemas de transporte ferroviarios frente a sistemas de transporte de autobuses equivalentes, es decir, que ofrecen al usuario exactamente las mismas condiciones en el desplazamiento considerado.

Existen diferentes enfoques para estudiar la capacidad de captación del transporte público, entre los que se pueden citar los estudios antes y después y los modelos de demanda basados en encuestas a los usuarios. En el caso del rail factor, al no estar basado en atributos claramente medibles, los aspectos psicológicos pasan a tener una gran relevancia (véase Anta, Novales, Conles y Orro, 2014).

En este trabajo se ha optado por el enfoque de estimación de modelos de elección discreta 
basado en encuestas de preferencias declaradas. Se han realizado diseños experimentales eficientes, basados en un diseño ortogonal previo aplicado a una encuesta piloto. Para una revisión del proceso de generación del diseño puede consultarse Anta, Novales, Orro y Conles (2014). Se han realizado encuestas en universidades de Francia y España, acometiéndose a continuación un proceso de selección del enfoque de modelización más adecuado.

La estructura de la ponencia es la siguiente: en el segundo apartado se presenta la encuesta realizada y las ciudades seleccionadas, en el tercero se expone lo referente a la especificación y estimación de modelos y en el cuarto se presentan los resultados obtenidos. Se finaliza con las conclusiones del estudio.

\section{LA ENCUESTA Y LA MUESTRA}

Tras una fase inicial en grupo focal y una encuesta piloto con diseño ortogonal, la encuesta se realizó con un diseño eficiente. Los atributos y niveles considerados pueden verse en la tabla $\mathrm{n}^{\circ}$ 1. Se consideraron desplazamientos típicos para cada una de las localizaciones estudiadas.

\begin{tabular}{|c|c|c|c|c|}
\hline ATRIBUTO & $\begin{array}{l}\text { ETIQUETA } \\
\text { ATRIBUTO }\end{array}$ & $\begin{array}{c}\text { CÓDIGO DISEÑO } \\
\text { NIVEL }\end{array}$ & $\begin{array}{c}\text { CÓDIGO } \\
\text { ORTOGONAL } \\
\text { NIVEL }\end{array}$ & $\begin{array}{l}\text { ETIQUETA } \\
\text { NIVEL }\end{array}$ \\
\hline \multirow{3}{*}{ Tiempo } & \multirow{3}{*}{$\mathrm{T}$} & 0 & -1 & Valor menor \\
\hline & & 1 & 0 & Valor intermedio \\
\hline & & 2 & 1 & Valor mayor \\
\hline \multirow{3}{*}{ Coste } & \multirow{3}{*}{$\mathrm{C}$} & 0 & -1 & Valor menor \\
\hline & & 1 & 0 & Valor intermedio \\
\hline & & 2 & 1 & Valor mayor \\
\hline \multirow{3}{*}{$\begin{array}{l}\text { Intervalo entre } \\
\text { Vehículos }\end{array}$} & \multirow{3}{*}{$\mathrm{F}$} & 0 & -1 & Valor menor \\
\hline & & 1 & 0 & Valor intermedio \\
\hline & & 2 & 1 & Valor mayor \\
\hline \multirow{3}{*}{$\mathrm{N}^{\mathrm{o}}$ Trasbordos } & \multirow{3}{*}{ NT } & 0 & -1 & Valor menor \\
\hline & & 1 & 0 & Valor intermedio \\
\hline & & 2 & 1 & Valor mayor \\
\hline \multirow{3}{*}{$\begin{array}{c}\text { Tipo de Transporte } \\
\text { Público }\end{array}$} & \multirow{3}{*}{ PT } & 0 & -1 & Definición Nivel C \\
\hline & & 1 & 0 & Definición Nivel B \\
\hline & & 2 & 1 & Definición Nivel A \\
\hline
\end{tabular}

Tabla 1. Atributos y niveles de atributo considerados en el diseño experimental eficiente para la encuesta final

Con el atributo tipo de transporte se pretende reflejar la calidad de servicio que ofrece el sistema, definiéndose en tres niveles, que se presentan en la figura 1. 


\begin{tabular}{|c|c|c|c|}
\hline CARACTERISTICAS & NIVEL A & NIVEL B & NIVEL C \\
\hline $\begin{array}{c}\text { Carril Reservado + Prioridad } \\
\text { en Intersecciones }\end{array}$ & $\begin{array}{c}\text { DISPONIBLE durante } \\
\text { todo el recorrido }\end{array}$ & $\begin{array}{c}\text { DISPONIBLE en la } \\
\text { MTAD del recorrido }\end{array}$ & $\begin{array}{c}\text { NO DISPONIBLE } \\
\text { en todo el recorrido }\end{array}$ \\
\hline $\begin{array}{c}\text { Información Suministrada } \\
\text { en las PARADAS }\end{array}$ & $\begin{array}{c}\text { TINERARIO+HORARIOS } \\
\text { de líneas } \\
\text { TIEMPO DE ESPERA en } \\
\text { tiempo real }\end{array}$ & $\begin{array}{c}\text { IDÉNTICA a la } \\
\text { NIVEL A }\end{array}$ & $\begin{array}{c}\text { TINERARIO+ } \\
\text { HORARIOS } \\
\text { de líneas }\end{array}$ \\
\hline $\begin{array}{c}\text { Aumento de la duración } \\
\text { prevista para 1 de cada 3 } \\
\text { desplazamientos }\end{array}$ & $0 \%$ (del tiempo total) & $15 \%$ (del tiempo total) & $40 \%$ (del tiempo total) \\
\hline
\end{tabular}

\section{Fig. 1. Presentación del atributo tipo de transporte público en la encuesta final}

Dentro de cada encuesta se definen dos escenarios posibles, que se diferencian en las alternativas disponibles en cada uno de ellos para realizar un desplazamiento tipo con una longitud predeterminada. En la versión final de la encuesta estos escenarios fueron los siguientes:

- Escenario A: solamente disponibles dos alternativas del transporte público, un sistema de transporte ferroviario y un sistema de transporte basado en autobús.

- Escenario B: además de los modos de transporte del escenario A, se incluye el automóvil privado como disponible para realizar el desplazamiento.

Adicionalmente, y con el objetivo de captar la influencia en la elección de ciertas variables relacionadas con la imagen percibida del transporte público, se han planteado dos experimentos diferentes a los encuestados: el experimento 1, en el que se considera el atributo tipo de transporte público como variable influyente en la elección; y el experimento 2 , en el que no se muestra este atributo y por tanto los encuestados no lo tienen en cuenta en sus elecciones.

Se seleccionaron áreas metropolitanas de tamaño intermedio por ser en ellas donde los sistemas de transporte analizados resultan más adecuados. La selección se realizó buscando diferentes grados de experiencia con LRT y BHLS. En España se seleccionó A Coruña, sin SCI pero con experiencia de un tranvía histórico, y Vitoria-Gasteiz, con metro ligero y red de autobús mejorado (sin llegar a ser un BHLS), siendo además European Green Capital en 2012. En Francia se seleccionaron Lille, con LRT, autobús mejorado y un sistema especial (VAL, vehículo automático ligero con plataforma independiente); Clermont-Ferrand y Valenciennes, ambas con LRT o sistemas análogos y autobús mejorado; y por último Nantes (European Green Capital en 2013), con red de LRT y BHLS. Las encuestas se realizaron en soporte papel en centros universitarios de las diferentes localizaciones seleccionadas. El número total de encuestas realizadas y de situaciones de elección válidas para la modelización se presenta en la tabla $n^{\circ} 2$. En el caso de A Coruña se realizaron dos encuestas, 
en la primera la etiqueta del modo ferroviario fue tranvía mientras que en la segunda fue metro ligero.

\begin{tabular}{|c|c|c|c|c|c|c|c|c|c|}
\hline \multirow{2}{*}{ LOCALIDAD } & \multicolumn{3}{|c|}{$\begin{array}{l}\text { № total de encuestas } \\
\text { realizadas }\end{array}$} & \multicolumn{3}{|c|}{$\begin{array}{l}\text { № de encuestas válidas } \\
\text { (observaciones PR) }\end{array}$} & \multicolumn{3}{|c|}{$\begin{array}{l}\text { № de situaciones de } \\
\text { elección válidas (PD) }\end{array}$} \\
\hline & Exp. 1 & Exp. 2 & Total & Exp. 1 & Exp. 2 & Total & Exp. 1 & Exp. 2 & Total \\
\hline A Coruña (1) & 243 & 248 & 491 & 227 & 233 & 460 & 3,545 & 3,699 & 7,244 \\
\hline A Coruña (2) & 275 & 274 & 549 & 253 & 254 & 507 & 2,553 & 2,708 & 5,261 \\
\hline Vitoria-Gasteiz & 146 & 136 & 282 & 115 & 114 & 229 & 1,767 & 1,750 & 3,517 \\
\hline Nantes & 81 & 65 & 146 & 79 & 60 & 139 & 1,363 & 1,052 & 2415 \\
\hline Lille & 167 & 167 & 334 & 156 & 159 & 315 & 2,618 & 2,693 & 5,311 \\
\hline Valenciennes & 79 & 68 & 147 & 68 & 55 & 123 & 1,155 & 933 & 2,088 \\
\hline $\begin{array}{c}\text { Clermont- } \\
\text { Ferrand } \\
\end{array}$ & 93 & 70 & 163 & 86 & 64 & 150 & 1,418 & 1,074 & 2,492 \\
\hline TOTAL & 339 & 305 & 2,112 & 310 & 278 & 1,923 & 5,191 & 4,700 & 28,328 \\
\hline
\end{tabular}

Tabla 2. Número de observaciones que forman las diferentes bases de datos

\section{MODELIZACIÓN}

Se ha buscado analizar las elecciones observadas en la muestra mediante el empleo de modelos de elección discreta basados en la teoría de la maximización de la utilidad aleatoria (para una revisión de este tipo de modelos puede verse Ben-Akiva y Lerman, 1985; Train, 2003 u Orro, 2006). Se adopta la hipótesis de que cada alternativa $j$ proporciona una utilidad $U_{j q}$ al individuo $q$ y que este escoge aquella alternativa que le reporta mayor utilidad. Desde el punto de vista del analista se considera una parte representativa de la utilidad $V_{n j}$, medible a partir de atributos $x$ del individuo, de la alternativa o de la situación de elección, ponderados por unos coeficientes $\beta$, a la que se añade un término aleatorio $\varepsilon$ que representa la diferencia entre la utilidad representativa y el valor real.

$$
U_{j q}=V_{j q}(\beta, x)+\varepsilon_{j q}
$$

Las hipótesis que se realicen acerca de la estructura del término aleatorio conducen a los diferentes modelos existentes en la literatura, de los que en este trabajo se han considerado el logit multinomial (MNL), el logit jerárquico (NL) y el logit mix to (ML).

La investigación presentada se ha caracterizado por una búsqueda sistemática y exhaustiva de la mejor especificación de la función de utilidad. Tomando como enfoque inicial el modelo logit multinomial y partiendo de los atributos incluidos en el diseño experimental, se ha establecido la siguiente especificación general: 


$$
\begin{aligned}
& V_{1}=\beta_{t, 1} \cdot t_{1}+\beta_{c, 1} \cdot c_{1} \\
& V_{2}=A S C_{2}+\beta_{t, 2} \cdot t_{2}+\beta_{c, 2} \cdot c_{2}+\beta_{f, 2} \cdot f_{2}+\beta_{n t 1,2} \cdot n t 1_{2}+\beta_{n t 2,2} \cdot n t 2_{2}+\beta_{p t B, 2} \cdot p t B_{2}+\beta_{p t A, 2} \cdot p t A_{2} \\
& V_{3}=A S C_{3}+\beta_{t, 3} \cdot t_{3}+\beta_{c, 3} \cdot c_{3}+\beta_{f, 3} \cdot f_{3}+\beta_{n t 1,3} \cdot n t 1_{3}+\beta_{n t 2,3} \cdot n t 2_{3}+\beta_{p t B, 3} \cdot p t B_{3}+\beta_{p t A, 3} \cdot p t A_{3}
\end{aligned}
$$

Siendo:

$j=1,2,3$ las alternativas disponibles (coche, autobús y modo ferroviario, respectivamente)

$V_{j}$ la utilidad sistemática o representativa de la alternativa $j$

$A S C_{j}$ la constate específica de la alternativa $j$

$\beta_{t, j}$ el coeficiente o parámetro correspondiente al tiempo de la alternativa $j$

$t_{j}$ el valor del tiempo para la alternativa $j$

$\beta_{c, j}$ el coeficiente o parámetro correspondiente al coste de la alternativa $j$

$c_{j}$ el valor del coste para la alternativa $j$

$\beta_{f, j}$ el coeficiente o parámetro correspondiente al intervalo entre vehículos de la alternativa $j$

$f_{j}$ el valor del intervalo entre vehículos para la alternativa $j$

$\beta_{n t l, j}$ el coeficiente o parámetro correspondiente a la categoría "1 trasbordo" para el atributo número de trasbordos de la alternativa $j$

$n t l_{j}$ el valor de codificación tipo effects correspondiente a la categoría "1 trasbordo" para el atributo número de trasbordos de la alternativa $j$

$\beta_{n t 2, j}$ el coeficiente o parámetro correspondiente a la categoría "2 trasbordos" para el atributo número de trasbordos de la alternativa $j$

$n t 2 j$ el valor de codificación tipo effects correspondiente a la categoría " 2 trasbordos" para el atributo número de trasbordos de la alternativa $j$

$\beta_{p t B, j}$ el coeficiente o parámetro correspondiente a la categoría "transporte público tipo B" para el atributo tipo de transporte público de la alternativa $j$

$p t B_{j}$ el valor de codificación tipo effects correspondiente la categoría "transporte público tipo B" para el atributo tipo de transporte público de la alternativa $j$

$\beta_{p t A, j}$ el coeficiente o parámetro correspondiente a la categoría "transporte público tipo A" para el atributo tipo de transporte público de la alternativa $j$

$p t A_{j}$ el valor de codificación tipo effects correspondiente la categoría "transporte público tipo A" para el atributo tipo de transporte público de la alternativa $j$

Se aprecia en la especificación mostrada que se toma al coche como alternativa de referencia para la identificación de las constantes específicas, y las categorías "ningún trasbordo" y "transporte público tipo C" como las de referencia para los atributos número de trasbordos y tipo de transporte público respectivamente.

Partiendo de esta especificación general se han desarrollado 25 especificaciones diferentes empleando restricciones de igualdad entre coeficientes. Una síntesis de estas especificaciones puede verse en la figura 2. Tras los análisis efectuados se ha tomado como 
especificación de referencia la número 13. Por otro lado se han estimado modelos logit multinomial que incluyen información adicional de cada individuo, como es la experiencia previa con la red de transporte público y la alternativa elegida en el desplazamiento indicado en la encuesta de preferencias reveladas. En total se han considerado 38 especificaciones MNL diferentes que se han estimado para los diferentes experimentos y escenarios, hasta llegar a 91 MNL por ubicación.

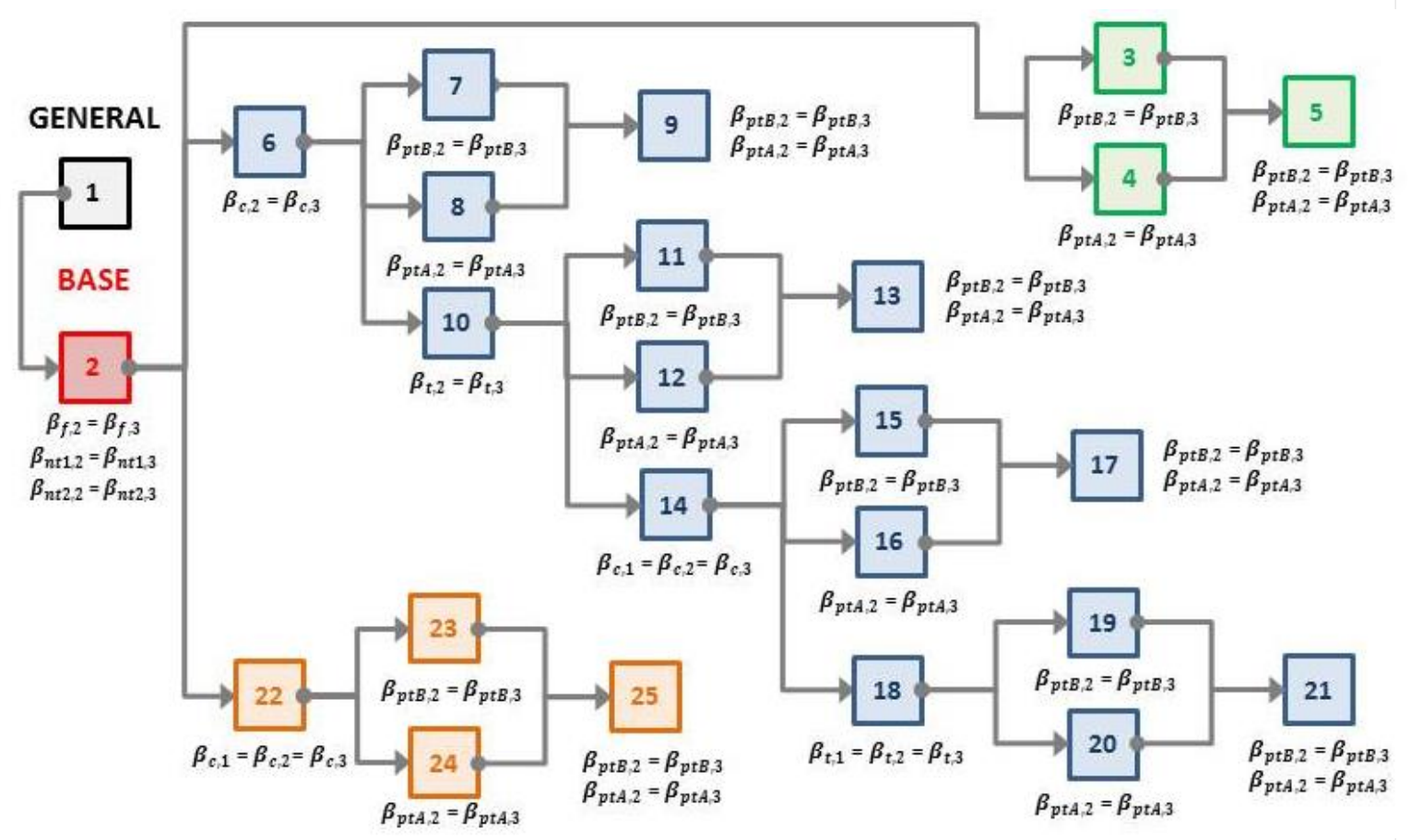

Fig. 2. Esquema de los diferentes modelos en función de las especificaciones de la utilidad de las alternativas a partir de la especificación base

En el marco de la investigación presentada, se han estimado también modelos logit jerárquico (únicamente para la elección entre las tres alternativas, 56 modelos NL por localización) y modelos logit mixto de dos tipos, el primero en especificación de componentes de error analizando las correlaciones entre alternativas y el segundo en la especificación de componentes aleatorios analizando variaciones en los gustos. Los modelos logit mixto han permitido tener en cuenta la correlación entre las diferentes respuestas del mismo encuestado. Se han estimado en total 140 modelos ML válidos en cada localización.

\section{RESULTADOS}

El número de resultados obtenidos en esta investigación es, evidentemente, muy elevado, realizándose 4970 modelizaciones (2989 para el experimento 1 y 1981 para el experimento 2 ). En esta ponencia se busca sintetizar unos primeros valores que permitan analizar la existencia o no de un rail factor. A lo largo de la investigación las dos alternativas se han denominado sistema ferroviario y sistema basado en autobús, pero a efectos de simplificar 
la exposición se denominarán en este documento LRT y BHLS respectivametne. La primera evidencia está en la elección entre LRT y BHLS a igualdad de condiciones en todos los atributos, que queda por tanto determinada por la constante específica. Se presentan únicamente resultados para las especificaciones que han resultado de mayor interés.

En la tabla 3 se muestran los resultados de las especificaciones seleccionadas para ambos experimentos en las diferentes ciudades analizadas. Se comienza analizando la ciudad de Nantes como caso emblemático en que existe experiencia de ambos sistemas. Para el experimento 2 ( $\sin$ considerar el tipo de transporte definido en la figura 1) y en el escenario A (sin disponibilidad de coche) los resultados correspondientes tanto al modelo MNL como al ML (que colapsa en el anterior por no detectarse variabilidad significativa) indican una ligera preferencia hacia el BHLS frente al LRT. Sin embargo, los test estadísticos muestran que esta diferencia no es significativa, por lo que en todo caso no se ha detectado en esas condiciones la existencia de una preferencia por los modos ferroviarios.

\begin{tabular}{|c|c|c|c|c|c|}
\cline { 3 - 6 } \multicolumn{2}{c|}{} & \multicolumn{2}{c|}{ Experimento 2 } & \multicolumn{2}{c|}{ Experimento 1 } \\
\cline { 3 - 6 } \multicolumn{2}{c|}{} & $\mathrm{P}_{\text {BHLS }}$ (media) & $\mathrm{P}_{\text {LRT }}$ (media) & $\mathrm{P}_{\text {BHLS }}$ (media) & $\mathrm{P}_{\text {LRT }}$ (media) \\
\hline \multirow{2}{*}{ Nantes } & $\mathrm{MNL}$ & $52.62 \%$ & $47.38 \%$ & $48.21 \%$ & $51.79 \%$ \\
\cline { 2 - 6 } & $\mathrm{ML}$ & $52.62 \%$ & $47.38 \%$ & $48.66 \%$ & $51.34 \%$ \\
\hline \multirow{2}{*}{ Clermont-Ferrand } & $\mathrm{MNL}$ & $46.66 \%$ & $53.34 \%$ & $39.22 \%$ & $60.78 \%$ \\
\cline { 2 - 6 } & $\mathrm{ML}$ & $47.26 \%$ & $52.74 \%$ & $39.48 \%$ & $60.52 \%$ \\
\hline \multirow{2}{*}{ Lille } & $\mathrm{MNL}$ & $49.44 \%$ & $47.38 \%$ & $48.21 \%$ & $51.79 \%$ \\
\cline { 2 - 6 } & $\mathrm{ML}$ & $52.62 \%$ & $47.38 \%$ & $48.66 \%$ & $51.34 \%$ \\
\hline \multirow{2}{*}{ Valenciennes } & $\mathrm{MNL}$ & $40.40 \%$ & $59.60 \%$ & $39.60 \%$ & $60.40 \%$ \\
\cline { 2 - 6 } & $\mathrm{ML}$ & $41.10 \%$ & $58.90 \%$ & $39.85 \%$ & $60.15 \%$ \\
\hline \multirow{2}{*}{ A Coruña (1) } & $\mathrm{MNL}$ & $69.17 \%$ & $30.83 \%$ & $69.06 \%$ & $30.94 \%$ \\
\cline { 2 - 6 } & $\mathrm{Tranvía}$ & $69.01 \%$ & $30.99 \%$ & $69.07 \%$ & $30.93 \%$ \\
\hline \multirow{2}{*}{ A Coruña (2) } & $\mathrm{MNL}$ & $61.28 \%$ & $38.72 \%$ & $62.71 \%$ & $37.29 \%$ \\
\cline { 2 - 6 } & $\mathrm{ML}$ & $61.37 \%$ & $38.63 \%$ & $62.79 \%$ & $37.21 \%$ \\
\hline \multirow{2}{*}{ Vitoria-Gasteiz } & $\mathrm{MNL}$ & $54.46 \%$ & $45.54 \%$ & $54.81 \%$ & $45.19 \%$ \\
\cline { 2 - 6 } & $\mathrm{ML}$ & $54.37 \%$ & $45.63 \%$ & $55.03 \%$ & $44.97 \%$ \\
\hline
\end{tabular}

Tabla 3. Probabilidad de elección de las alternativas de transporte público

\section{suponiendo igualdad de condiciones. Escenario A.}

En el experimento 1 se incluye el atributo del tipo de transporte, derivado de la realización del grupo focal, encuesta piloto y otras fuentes bibliográficas, y que puede considerarse un indicador de las principales características que los usuarios asocian con la imagen de un SCI. Prestando únicamente atención al valor de la constante específica, se ha obtenido que no es significativamente distinta de cero, lo que supone que no existe una preferencia intrínseca por ninguno de los dos modos. Atendiendo al valor estimado se observaría una ligera preferencia por el modo ferroviario. En el caso de los modelos logit mixto sí se ha apreciado una variabilidad significativa en las preferencias por uno u otro modo dentro de la población, 
como puede apreciarse en la figura 3.

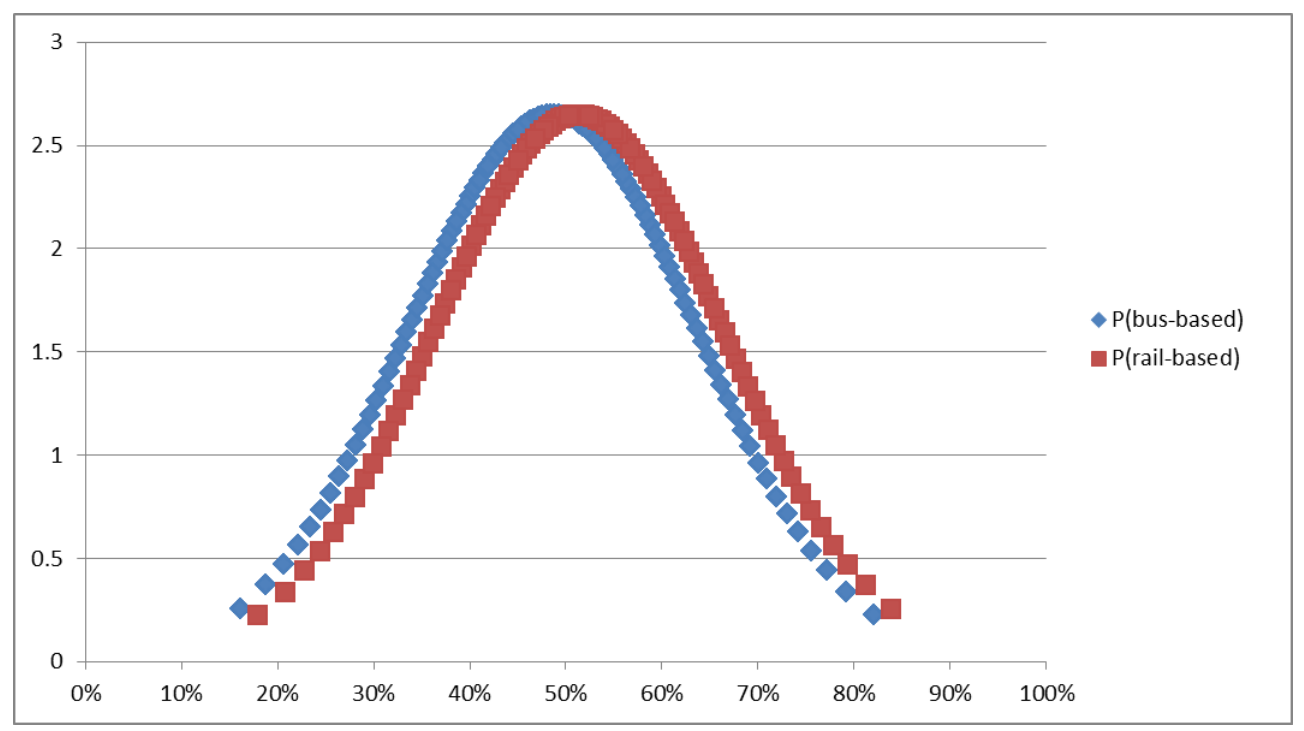

Fig. 3. Distribución de la probabilidad de elección según ML(EC) de las alternativas de transporte público suponiendo igualdad de condiciones: Exp. 1 / Esc. A, Nantes

Analizando las restantes ciudades, se puede apreciar que no existe una preferencia unánime ni hacia el BHLS ni hacia el LRT, por lo que no puede concluirse que el rail factor exista de forma generalizada. Algunas ciudades como Valenciennes muestran una preferencia clara por el modo ferroviario mientras que en otras como A Coruña la preferencia es clara por el autobús. En el caso de A Coruña la influencia de la experiencia de un tranvía histórico que no funcionaba propiamente como modo de transporte urbano puede tener relevancia, de hecho, la modificación de la etiqueta de tranvía a metro ligero supone una clara mejora en la valoración del modo ferroviario, pero sin invertir la preferencia por el autobús. Esto evidencia cómo la imagen percibida de los sistemas influye en las elecciones declaradas.

\section{CONCLUSIONES}

En la presente investigación se ha realizado un análisis experimental de la existencia del rail factor (preferencia intrínseca por el modo ferroviario) en diversas ciudades de tamaño medio de España y Francia. El estudio se basa en encuestas de preferencias reveladas y declaradas, obtenidas mediante un diseño eficiente y realizadas a estudiantes universitarios. Tras un intenso trabajo de modelización, se han seleccionado las especificaciones que se han encontrado más adecuadas para reflejar las elecciones estudiadas.

Puede concluirse de este análisis que no puede afirmarse que exista de forma generalizada una preferencia de la población analizada hacia un modo ferroviario frente a un sistema de autobuses con las mismas prestaciones. El modo preferido a igualdad de valores de los atributos presentados es diferente dependiendo de la población estudiada, los sistemas de 
transporte existentes en la ciudad o la experiencia previa de los usuarios. Por ello, se recomienda realizar encuestas en las condiciones locales cuando sea preciso evaluar la captación potencial de un nuevo sistema en estudio, sin que sea adecuado extrapolar las preferencias observadas en otros lugares.

En el marco de la investigación se han realizado además otros análisis y simulaciones para estudiar en mayor profundidad la influencia de los factores reflejados en la encuesta de preferencias reveladas y la potencial captación de viajeros en caso de implantar uno u otro sistema.

\section{AGRADECIMIENTOS}

El presente documento ha sido llevado a cabo por investigadores miembros del Grupo de Ferrocarriles y Transportes perteneciente a la Universidad de A Coruña. Está enmarcado en el proyecto de investigación NASCI: “Comparación tecnológica, económica y de captación de usuarios de los Nuevos Avances en Sistemas de transporte metropolitano de Capacidad Intermedia", que ha sido financiado por el Ministerio de Economía y Competitividad dentro del Plan Nacional de I+D+i 2008-2011.

\section{REFERENCIAS}

ANTA, J., NOVALES, M., CONLES, E. y ORRO, A. (2014) Influencia de los aspectos psicológicos en la elección de modo de transporte: modos ferroviarios frente al autobús con alto nivel de servicio. XI Congreso de Ingeniería del Transporte. Santander, 9 al 11 de junio de 2014

ANTA, J., NOVALES, M., ORRO, A. y CONLES, E. (2014) Diseños experimentales eficientes: revisión metodológica y aplicación a un caso de estudio para la captación de viajeros en sistemas de transporte metropolitano de capacidad intermedia. XVIII Congreso Panamericano de Ingeniería de Tránsito, Transporte y Logística. Santander, 11 al 13 de junio de 2014.

AXHAUSEN, K. W., HAUPT, T., FELL, B. y HEIDL, U. (2001). Searching for the rail bonus: Results from a panel SP/RP study. European Journal of Transport and Infrastructure Research, 1(4), 353-369.

BEN-AKIVA, M. y S.R. LERMAN (1985) Discrete Choice Analysis. Theory and Application to Travel Demand, The MIT Press, Cambridge, Massachusetts.

BUNSCHOTEN, T. (2012). To tram or not to tram: Exploring the existence of the tram bonus. Graduation thesis, Delft University of Technology. 
NOVALES, M., ORRO, A., CONLES, E. y ANTA, J. (2014) Medium-capacity transit systems. Some reflections about making the right choice. En Pratelli A. Public Mobility Systems, WITPress, Southampton (UK), 2014. ISBN: 978-1-84564-908-1

ORRO, A. (2006) Modelos de elección discreta en transportes con coeficientes aleatorios, Cátedra Abertis, Barcelona.

PICKRELL, D.H. (1992). A Desired Named Streetcar: Fantasy and Fact in Rail Transit Planning. Journal of the American Planning Association, 58 (2), 158-176.

SCHERER, M. (2011). The image of bus and tram: first results. 11th Swiss Transport Research Conference. May 11-13, 2011. Monte Veritá, Ascona.

TRAIN, K. (2003) Discrete Choice Methods with Simulation, Cambridge University Press, Cambridge. 